Artigos Originais

\title{
Doença celiaca e infertilidade feminina: associação freqüentemente negligenciada
}

\author{
Celiac disease and female infertility: a frequently neglected association \\ Carmen Livia da Silva Martins ${ }^{1}$, Lenora Gandolfi², Pedro Luiz Tauil ${ }^{3}$, \\ Marilúcia de Almeida Rocha Picanço ${ }^{4}$, Maria Ophelia Galvão de Araujo ${ }^{5}$, Riccardo Pratesi ${ }^{6}$
}

\section{RESUMO}

Objetivo: Verificar a existência de associação entre subfertilidade ou infertilidade e concomitante presença de doença celíaca nas mulheres atendidas em ambulatório de hospital geral especializado em reprodução humana. Métodos: O delineamento do estudo foi do tipo caso-controle. Os casos foram constituídos por um grupo de 200 mulheres com queixa de dificuldade para engravidar, e um grupo controle formado por 400 mulheres atendidas no mesmo ambulatório com queixas variadas, mas sem problemas de fertilidade. Ambos os grupos de pacientes foram submetidos a pesquisa de anticorpos antiendomísio pelo método de imunofluorescência indireta. Nos casos positivos, o diagnóstico foi confirmado por biópsia duodenal endoscópica e exame histopatológico. Resultados: Os testes antiendomísio no grupo com dificuldade para engravidar resultaram positivos em três pacientes $(1,5 \%)$. O diagnóstico de doença celíaca foi confirmado por subseqüente exame histopatológico da mucosa duodenal. Todas as pacientes pertencentes ao grupo controle apresentaram testes sorológicos negativos. Conclusões: $\mathrm{O}$ fato de terem sido encontrados casos de doença celíaca somente entre mulheres com queixas de dificuldade para engravidar parece um dado relevante e sugestivo de ser a doença celíaca mais comum entre mulheres inférteis; porém, há necessidade de novos estudos com tamanho de amostra maior para confirmação definitiva dessa provável associação.

PALAVRAS-CHAVE: Doença celíaca; Infertilidade feminina; Estudos de casos e controles

\section{ABSTRACT}

Purpose: To verify the existence of association between sub-fertility or infertility and concurrent celiac disease in women attended at a reproductive disorders out-patient clinic of a general hospital. Methods: This was a case-control study in which a group of 200 women with difficulty in conceiving was compared with a control group with 400 women who were treated at the same hospital for various reasons, but not for fertility problems. Both groups were submitted to the anti-endomysium antibody protocol using the indirect immunofluorescence method. Whenever positive, the diagnosis was further confirmed by endoscopic duodenal biopsy and histopathological exams. Results: Anti-endomysium testing resulted positive in three patients $(1.5 \%)$ within the group of women with difficulty in conceiving. Diagnosis of celiac disease was subsequently confirmed by histopathological exams of the duodenal mucosa. All the control patients presented negative results in the serological exams. Conclusions: Celiac disease was observed only in women with difficulty in conceiving, a relevant fact indicating that this disease may be more common in infertile women. However, new studies with a greater sample size are needed to confirm this possible association.

KEYWORDS: Celiac disease; Infertility, female; Case-control studies

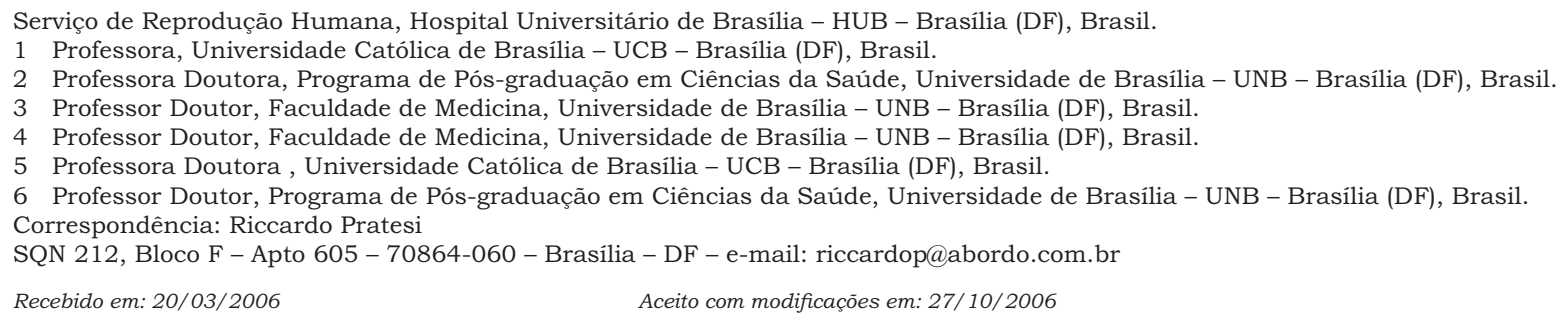




\section{Introdução}

A doença celíaca (DC) é uma afecção inflamatória crônica caracterizada por permanente intolerância ao glúten contido no trigo e em cereais afins. Em sua forma clássica manifesta-se por diarréia, distensão abdominal e desnutrição progressiva. Estas alterações, em indivíduos geneticamente predispostos resultam de resposta auto-imune mediada por linfócitos T, que leva a lesão progressiva no intestino delgado, caracterizada por infiltração linfocitária no epitélio jejunal, atrofia vilositária e hipertrofia nas criptas. Tanto as manifestações clínicas como as lesões do intestino tendem à normalização com a introdução de rigorosa dieta sem glúten ${ }^{1}$. Do ponto de vista epidemiológico a DC configura-se como importante problema de saúde pública. Embora até poucas décadas atrás fosse considerada uma afecção quase exclusivamente confinada a países europeus, tem sido detectada também em outros continentes com prevalência muito similar à encontrada na Europa, onde recentes estudos de rastreamento evidenciaram prevalência variando entre 0,5 e $2 \%^{2}$. No Brasil, onde era considerada uma doença tradicionalmente rara, estudos de rastreamento detectaram uma prevalência variando entre 1:681 em grupo de doadores de sangue presumivelmente sadios ${ }^{3}$ e 1:293 em grupo de adultos e crianças sem queixas gastroentéricas ${ }^{4}$.

Além das lesões intestinais e a conseqüente sindrome de má absorção, a DC apresenta amplo espectro de sintomas e de afecções associadas que englobam vários outros órgãos e sistemas, freqüentemente sem sintomas gastroentéricos importantes. Embora a diarréia ainda seja a queixa inicial mais citada, outros eventos freqüentemente observados são: anemia ferropriva resistente ao tratamento, osteoporose, baixa estatura, dores articulares e dor abdominal recorrente. Dentre as afecções que constituem grupo de risco para concomitante presença de DC podem-se citar diabetes tipo 1, síndrome de Down, sindrome de Turner, além de síndromes neurológicas como epilepsia com calcificação occipital, ataxia cerebelar e neuropatia periférica ${ }^{5}$.

Controvérsias ainda existem com relação a possivel associação entre a DC e disfunção do aparelho reprodutor feminino. Vários estudos sugeriram que antes da instalação de dieta sem glúten mulheres com DC teriam maior prevalência de abortos recorrentes, crescimento intra-uterino restrito e natimortalidade aumentada ${ }^{6-13}$. A hipótese de que a DC poderia ser considerada um fator de risco plausivel para o aparecimento de malformações congênitas também foi aventada, pois as lesões jejunais levam a uma síndrome de má absorção com conseqüente carência de nutrientes como ferro, zinco e vitaminas (principalmente ácido fólico e vitaminas $B_{12}, \mathrm{~K}$ e $\mathrm{B}_{6}$ ), elementos importantes para o desenvolvimento fetal normal. No entanto, estudos epidemiológicos adequados enfocando incidência de malformações congênitas em filhos de mulheres com DC são praticamente inexistentes, afora um estudo isolado ${ }^{14}$. Este estudo apresentou um único caso de defeito de fechamento de tubo neural entre os filhos de 60 mães com DC $(1,6 \%)$. Apesar de a prevalência encontrada ser superior à esperada para a mesma região geográfica, a limitação do número de casos não permitiu uma validação estatística deste achado. Pelo contrário, outros estudos enfocando desordens do aparelho reprodutor feminino em mulheres com DC evidenciaram uma associação pouco significativa ou mesmo praticamente nula entre eventos adversos durante a gravidez e presença de $\mathrm{DC}^{15-17}$.

Dúvidas similares também existem quando se enfoca especificamente a queixa de subfertilidade ou infertilidade. Vários estudos têm consistentemente apontado para uma vida reprodutiva mais curta, com menarca tardia e menopausa precoce em mulheres com $\mathrm{DC}^{6,9,12,18-22}$.

No atual estado de conhecimento a possivel influência deletéria da concomitante presença de DC sobre o sistema reprodutor feminino não está ainda definitivamente confirmada.

O objetivo do presente estudo foi o de verificar a existência de associação entre subfertilidade ou infertilidade e concomitante presença de DC em mulheres atendidas em ambulatório especializado em reprodução humana de hospital geral, sendo a DC identificada por meio da pesquisa de presença de anticorpos antiendomísio e confirmada por biópsia jejunal por via endoscópica.

\section{Métodos}

Este é um estudo epidemiológico do tipo caso-controle de base ambulatorial. Foram seqüencialmente incluídas na pesquisa 200 pacientes que procuraram espontaneamente o ambulatório do Serviço de Reprodução Humana do Hospital Universitário de Brasília com queixa de dificuldade para engravidar, obedeceram aos critérios de inclusão e consentiram em participar do estudo (desenvolvido de setembro de 2002 a setembro de 2003). O grupo controle foi formado por $400 \mathrm{mu}-$ lheres com idades similares às do grupo em estudo, que também procuraram espontaneamente o ambulatório de ginecologia e obstetrícia do mesmo hospital com vários motivos para consulta, mas 
sem queixa de infertilidade. A pesquisa foi aprovada pelo Comitê de Ética em Pesquisa da Faculdade de Ciências da Saúde da Universidade de Brasília e tanto as pacientes como os controles assinaram termo de consentimento esclarecendo os objetivos e possiveis riscos e beneficios da pesquisa.

Os critérios de inclusão no grupo de casos foram: dificuldade para engravidar por período igual ou maior que um ano e idade compreendida entre 16 e 45 anos. Nenhuma investigação prévia para determinar a possivel causa da infertilidade havia sido efetuada nas pacientes incluidas no estudo; no entanto, foi afastada a possibilidade de esta ser atribuivel ao cônjuge. Amostras de sangue venoso foram obtidas de todas as mulheres admitidas no estudo (pacientes e controles). Após centrifugação o soro resultante foi estocado a $-20^{\circ}$ até o momento de seu uso.

O rastreamento de casos positivos foi efetuado utilizando-se uma pesquisa de anticorpos antiendomísio da classe IgA (IgA-EMA) pelo método de imunofluorescência indireta, utilizando-se um corte criostático do terço distal de esôfago de primata como substrato ${ }^{23}$. Como segundo anticorpo foi utilizada anti-imunoglobulina humana da classe IgA conjugada com isotiocianato de fluoresceína (FITC) (ByoSystem S.A., Barcelona, Espanha). As lâminas foram examinadas por dois pesquisadores independentes, sendo considerados positivos os testes que apresentaram padrão reticular verde brilhante característico (em "favo de mel") na camada muscular lisa do esôfago ao exame em microscópio de fluorescência.

Foi realizada biópsia jejunal por via endoscópica nas pacientes que apresentaram teste sorológico positivo, tendo sido obtidas três a quatro amostras de mucosa duodenal, que foram submetidas a procedimento de rotina. As lâminas coradas pelo método de hematoxilina-eosina foram examinadas independentemente por patologista e gastroenterologista com experiência prévia em DC. O diagnóstico histopatológico baseou-se na presença de linfócitos intra-epiteliais (LIEs), hiperplasia de criptas e atrofia das vilosidades. Os resultados foram classificados de acordo com os critérios de $\mathrm{Marsh}^{24} \mathrm{em}$ : tipo 0 (normal), onde se observa mucosa sem alteração, um número de LIEs < 40 para cada 100 células do epitélio; tipo 1 (infiltrativo), caracterizado por vilosidades com arquitetura normal, com aumento nos LIEs > 40; tipo 2 (hiperplásico), caracterizado por aumento nos LIEs, arquitetura normal das vilosidades e hipertrofia nas criptas; tipo 3 (destrutivo), em que, além do aumento nos LIEs e hipertrofia das criptas, são observados diferentes graus de hipotrofia nas vilosidades, que pode variar de moderada a severa, podendo chegar a atrofia total.

\section{Resultados}

A idade das 200 pacientes com queixa de infertilidade variou entre 15 e 45 anos, com idade média de 28,6 anos e desvio padrão de 5,9. Neste grupo, três pacientes apresentaram teste IgA-EMA positivo e foram submetidas a biópsia duodenal endoscópica, que confirmou o diagnóstico de DC. O grau de alteração histopatológica nas amostras de mucosa duodenal destas pacientes, de acordo com a classificação de $\mathrm{Marsh}^{24}$, pode ser visto na Tabela 1 . A prevalência de DC neste grupo foi de 1:66 (1,5\%). Em nenhuma das pacientes diagnosticadas com DC foi detectada história de aborto. Em média, o tempo decorrido entre o início da queixa de dificuldade para engravidar e o diagnóstico de DC foi de 4,6 anos. As principais características das três pacientes com DC estão apresentadas na Tabela 1.

A idade das 400 pacientes que formaram o grupo controle também variou entre 15 e 45 anos, com idade média de 28,5 anos e desvio padrão de 7,6 . Neste grupo não foi detectado nenhum teste sorológico positivo para DC. Por esta razão não foi possivel calcular a medida de associação (razão de odds).

Tabela 1 - Principais características de pacientes com diagnóstico confirmado de doença celíaca.

\begin{tabular}{|c|c|c|c|c|c|}
\hline Pacientes & Idade (anos) & Gestações prévias & Filhos & Sintomas & Resultados de biópsiaa \\
\hline FOS & 24 & 0 & 0 & $\begin{array}{l}\text { Distensão abdominal, flatulência, } \\
\text { aftas recidivantes }\end{array}$ & Marsh II \\
\hline $\mathrm{CL}$ & 26 & 1 & 1 & $\begin{array}{l}\text { Perda de peso há um ano, anemia persis- } \\
\text { tente, dores articulares, constipação, apatia }\end{array}$ & Marsh IIIc \\
\hline AMS & 25 & 0 & 0 & $\begin{array}{l}\text { Baixo peso corporal, aftas recidivantes, dor } \\
\text { abdominal recorrente, constipação, apatia }\end{array}$ & Marsh III \\
\hline
\end{tabular}




\section{Discussão}

Apesar de significativo avanço diagnóstico das causas de infertilidade e subfertilidade, a dificuldade ou incapacidade para conceber permanece inexplicada em 25 a 30\% dos casais; cerca de 10 a $15 \%$ deles concebem um número menor de filhos do que desejariam, procurando especialistas na área de reprodução humana pelo menos uma vez na vida ${ }^{25}$

A questão da existência de associação entre DC e presença de subfertilidade e infertilidade ainda é controversa, mas vários estudos apontam para uma ligação entre sensibilidade ao glúten e desordens reprodutivas na mulher. Mulheres com DC apresentam menarca tardia e menopausa precoce e maior prevalência de amenorréia secundária ${ }^{8,9}$. Além disso, mesmo nos casos em que a gravidez é obtida, vários estudos têm demonstrado que em mulheres com DC não tratadas a taxa de aborto espontâneo é maior que a encontrada na população geral ${ }^{8}$; nestes casos o risco relativo de aborto múltiplo e recém-nato de baixo peso é 8,9 vezes maior do que o da população geral ${ }^{10}$.

Prevalência aumentada de DC entre mulheres inférteis foi documentada por diversos autores $^{12,18,19}$ e estima-se que a DC afete entre 4 e $8 \%$ das mulheres com diagnóstico de infertilidade sem causa aparente ${ }^{18}$. Como já foi citado, em grupo de mulheres inférteis sem investigação prévia tentando esclarecer a causa da infertilidade, a prevalência encontrada variou entre $1,42^{12} \mathrm{e}$ $3,03 \%{ }^{19}$. Por outro lado, um estudo do tipo casocontrole não evidenciou aumento significativo na prevalência de DC entre mulheres inférteis ${ }^{15}$. No entanto, é importante notar que neste estudo tanto o número de pacientes como o número de controles era relativamente pequeno, o que possivelmente não permitiu avaliar a significância dos resultados. Já um estudo de coorte histórica, bem mais amplo e significativo (base de dados do sistema assistencial de saúde do Reino Unido, englobando 1.521 mulheres com DC e 7.732 sem DC) concluiu que, exceto uma propensão à gravidez mais tardia, a fertilidade das mulheres com DC seria similar à dos controles. Haveria uma tendência para menor fertilidade naquelas com DC mais jovens, sendo que esta diferença tenderia a ser compensada em idade mais avançada ${ }^{16}$. Apesar do número impressionante de indivíduos estudados, o trabalho apresenta o viés próprio de estudos cujos dados foram colhidos em prontuários, que nem sempre expressam à verdade.

O mecanismo subjacente a estas desordens não foi ainda esclarecido e alguns autores advogam como provável causa a má nutrição e possíveis deficiências de zinco, ferro e folato ${ }^{26}$. No entanto, é importante lembrar que a infertilidade pode ocorrer na ausência de desnutrição evidente, podendo ser o único sintoma presente em pacientes com DC subclínica não diagnosticada ${ }^{18}$.

Em recente pesquisa chamou-se atenção para a evidente correlação entre o tempo de exposição ao glúten e o progressivo aumento de desordens auto-imunes ${ }^{27}$. A presença de auto-imunidade em indivíduos com DC pode variar entre extremos de $5,1 \%$ entre crianças com menos de dois anos e $34 \%$ em adultos com mais de 20 anos de ida$\mathrm{de}^{28}$. Estes autores aventam a possibilidade de que presença de anticorpos específicos e resposta auto-imune mediada por células $\mathrm{T}$ poderiam estar associadas à eclosão de disfunção do aparelho reprodutor feminino.

Em nosso estudo a prevalência de DC entre mulheres com dificuldade para engravidar foi de 1,5\% (3:200), com $\mathrm{IC}_{95 \%}$ de 0 a 3,2\%. Nenhum caso de DC foi identificado entre as mulheres do grupo controle. O resultado encontrado aproxima-se muito dos achados em estudos previamente citados, todos efetuados na Europa ${ }^{8,12,18-20}$. O resultado torna-se mais significativo se considerarmos a variabilidade do patrimônio genético da população brasileira, em que está presente um considerável aporte das raças negra e ameríndia. Apesar da alta prevalência em populações de origem árabe na África do Norte ${ }^{29}$, a DC ainda não foi documentada na raça negra ${ }^{30}$, e como sua existência entre nossos indigenas nunca foi investigada, é ainda uma incógnita.

Todas as pacientes diagnosticadas com DC apresentaram sintomatologia atípica, tendo poucos sintomas relacionados com o sistema gastroentérico. Diarréia (eventual ou freqüente), o sintoma mais comumente associado a DC, não foi referida por nenhuma das pacientes; contrariamente, duas queixaram-se de constipação. A não rara atipia dos sintomas e a freqüente paucidade dos sintomas gastroentéricos fazem da DC uma das afecções em que mais tempo decorre entre o inicio dos sintomas e o diagnostico final. Já na década de 80 se observava que decorriam em média 7 anos entre o aparecimento dos primeiros sintomas inespecíficos e o diagnóstico final de $\mathrm{DC}^{31}$. Desde então, talvez devido à maior percepção da existência de DC atípica, este tempo diminuiu razoavelmente, e um estudo multicêntrico realizado na Alemanha em 1996 assinalava uma demora média de 5,4 anos entre os primeiros sintomas da doença e o diagnóstico definitivo ${ }^{32}$.

O seguimento das pacientes diagnosticadas com DC revelou interessantes dados. Três a

Rev Bras Ginecol Obstet. 2006; 28(10): 601-6. 
quatro anos após o diagnóstico de DC, uma das pacientes informou nunca ter conseguido seguir dieta sem glúten, continuando sem conseguir engravidar. Seus testes sorológicos foram repetidos mais duas vezes e continuaram positivos. A segunda paciente (que já tinha tido um filho após gravidez sem intercorrência) teve um segundo filho e confessou (em recente contato telefônico) que, embora tenha diminuído a quantidade de glúten ingerido, nunca conseguiu seguir uma dieta estrita. Testes sorológicos de controle não foram efetuados nessa paciente, pois ela não compareceu às consultas de seguimento. A terceira paciente informou ter seguido estrita dieta isenta de glúten desde o diagnóstico da DC, o que foi confirmado por repetidos testes sorológicos de controle negativos; esta paciente engravidou aproximadamente um ano após o início da dieta e deu à luz uma criança normal.

O seguimento dessas pacientes evidencia uma das maiores dificuldades no tratamento da DC, ou seja, o da manutenção de uma dieta isenta de glúten. Mesmo tendo como importante motivação a possivel maternidade, duas das três pacientes positivas não conseguiram ou não quiseram seguir a dieta. Falta de adesão à dieta é freqüente entre as pessoas com DC, principalmente entre adolescentes e adultos jovens ${ }^{33}$.

É importante lembrar que DC não pode ser mais considerada doença restrita ao âmbito da gastroenterologia; pelo contrário, pode ser detectada em pacientes das mais diversas especialidades médicas e deve sempre ser ativamente pesquisada. O diagnóstico de DC é dificil, pois a maioria dos pacientes adultos apresenta formas atípicas, com escassos ou até mesmo nenhum sintoma observável. Muitos pacientes somente se queixam de um mal-estar indefinivel e inespecífico; em conseqüência, é provável que haja na comunidade um grande número de casos não diagnosticados. Mesmo em países desenvolvidos, estima-se que haja de 5 a 10 casos não diagnosticados para cada caso identificado ${ }^{30}$. Neste contexto, o aforismo "doença celíaca, se pensar nela você a encontrará" parece bastante apropriado. $\mathrm{Na}$ esfera da ginecologia e obstetrícia, contrabalançando importantes estudos que apontam falta de evidência concreta de possiveis efeitos deletérios da DC sobre o aparelho reprodutor feminino ${ }^{16,17}$, há vários estudos evidenciando baixa fertilidade ou mesmo infertilidade e maior prevalência de eventos desfavoráveis durante e gravidez em mulheres com DC6-13,17-21.

Diante do exposto e devido ao baixo custo dos testes que atualmente fazem parte dos exames laboratoriais de rotina, consideramos justificável o rastreamento de possiveis casos de DC entre mulheres com queixa de infertilidade, especialmente porque a investigação de possíveis causas de infertilidade geralmente é demorada, trabalhosa e cara, redundando eventualmente em resultado inconclusivo. O diagnóstico precoce de DC nestas pacientes evitaria investigações demoradas, desnecessárias e custosas na busca de uma possivel causa para sua infertilidade.

\section{Referências}

1. Stepniak D, Koning F. Celiac disease - sandwiched between innate and adaptative immunity. Hum Immunol. 2006;67(6):460-8.

2. Rewers M. Epidemiology of celiac disease: what are the prevalence, incidence, and progression of celiac disease? Gastroenterology. 2005;128(4 Suppl 1): S47-51.

3. Gandolfi L, Pratesi R, Córdoba JC, Tauil PL, Gasparin M, Catassi C. Prevalence of celiac disease among blood donors in Brazil. Am J Gastroenterol. 2000;95(3):689-92.

4. Pratesi R, Gandolfi L, Garcia SG, Modelli IC, Lopes de Almeida A, Bocca AL, et al. Prevalence of coeliac disease: unexplained age-related variation in the same population. Scand J Gastroenterol. 2003;38(7):747-50.

5. Green HR, Jabri B. Celiac disease. Annu Rev Med. 2006;57:207-21.

6. Ferguson R, Holmes GK, Cooke WT. Coeliac disease, fertility, and pregnancy. Scand J Gastroenterol. 1982;17(1):65-8.

7. Molteni N, Bardella MT, Bianchi PA. Obstetric and gynecological problems in women with untreated celiac sprue. J Clin Gastroenterol. 1990;12(1):37-9.

8. Sher KS, Mayberry JF. Female fertility, obstetric and gynaecological history in coeliac disease: a case control study. Acta Paediatr Suppl. 1996;412:76-7.

9. Smecuol E, Maurino E, Vazquez H, Pedreira S, Niveloni S Mazure R, et al. Gynaecological and obstetric disorders in coeliac disease: frequent clinical onset during pregnancy or the puerperium. Eur $\mathrm{J}$ Gastroenterol Hepatol. 1996;8(1):63-89.

10.Ciacci C, Cirillo M, Auriemma G, Di Dato G, Sabbatini F, Mazzacca G. Celiac disease and pregnancy outcome. Am J Gastroenterol.1996;91(4):718-22.

11.Norgard B, Fonager K, Sorensen HT, Olsen J. Birth outcomes of women with celiac disease: a nationwide historical cohort study. Am J Gastroenterol. 1999;94(9)2435-40.

12. Martinelli P, Troncone R, Paparo F, Torre P, Trapanese E, Fasano C, et al. Coeliac disease and unfavorable outcome of pregnancy. Gut. 2000;46(3):332-5. 
13. Gasbarrini A, Sanz Torre E, Trivellini C, De Carolis S, Caruso A, Gasbarrini G. Recurrent spontaneous abortion and intrauterine fetal growth retardation as symptoms of coeliac disease. Lancet. 2000;356(9227):399-400.

14.Dickey W, Stewart F, Nelson J, McBreen G, McMillan SA, Porter KG. Screening for coeliac disease as a possible maternal risk factor for neural tube defect. Clin Genet. 1996;49(2):107-8.

15.Kolho KL, Tiitinen A, Tulppala M, Unkila-Kallio L, Savilahti E. Screening for coeliac disease in women with history of recurrent miscarriage or infertility. $\mathrm{Br}$ J Obstet Gynaecol. 1999;106(2):171-3.

16.Tata LJ, Card TR, Logan RF, Hubbard RB, Smith CJ, West J. Fertility and pregnancy-related events in women with celiac disease: a population-based cohort study. Gastroenterology. 2005;128(4):849-55.

17. Greco L, Veneziano A, Di Donato L, Zampella C, Pecoraro M, Paladini D, et al. Undiagnosed coeliac disease does not appear to be associated with unfavourable outcome of pregnancy. Gut. 2004;53 (1):149-51.

18.Collin P, Vilska S, Heinonen PK, Hällström O, Pikkarainen P. Infertility and coeliac disease. Gut. 1996:39(3):382-4.

19.Meloni GF, Dessole S, Vargiu N, Tomasi PA, Musumeci $\mathrm{S}$. The prevalence of coeliac disease in infertility. Hum Reprod. 1999;14(11)2759-61.

20.Stazi AV, Mantovani A. A risk factor for female fertility and pregnancy: celiac disease. Gynecol Endocrinol. 2000;14(6):454-63.

21. Shamaly H, Mahameed A, Sharony A, Shamir R. Infertility and celiac disease: do we need more than one serological marker? Acta Obstet Gynecol Scand. 2004;83(12):1184-8.

22.Tiboni GM, de Vita MG, Faricelli R, Giampietro F, Liberati M. Serological testing for celiac disease in women undergoing assisted reproduction techniques. Hum Reprod. 2006;21(2):376-9.

23. Chorzelski TP, Beutner EH, Sulej J, Tchorzewska H, Jablonska S, Kumar V, et al. IgA anti-endomysium antibody. A new immunological marker of dermatitis herpetiformis and coeliac disease. Br J Dermatol. 1984;111(4):395-402.

24. Marsh MN. Gluten, major histocompatibility complex, and the small intestine. A molecular and immunobiologic approach to the spectrum of gluten sensitivity ('celiac sprue'). Gastroenterology. 1992;102(1): 330-54.

25. Evers JL. Female subfertility. Lancet. 2002;360 (9327):151-9.

26.Rostami K, Steegers EA, Wong WY, Braat DD, Steegers-Theunissen RP. Coeliac disease and reproductive disorders: a neglected association. Eur J Obstet Gynecol Reprod Biol. 2001;96(2):146-9.

27.Bustos D, Moret A, Tambutti M, Gogorza S, Testa $\mathrm{R}$, Ascione A, et al. Autoantibodies in Argentine women with recurrent pregnancy loss. Am J Reprod Immunol. 2006;55(3):201-7

28.Ventura A, Magazzu G, Greco L. Duration of exposure to gluten and risk for autoimmune disorders in patients with celiac disease. SIGEP Study Group for Autoimmune Disorders in Celiac Disease. Gastroenterology. 1999;117(2):297-303.

29. Catassi C, Rätsch IM, Gandolfi L, Pratesi R, Fabiani E, El Asmar R, et al. Why is coeliac disease endemic in the people of the Sahara? Lancet. 1999;354(9179): 647-8.

30. Cataldo F, Lio D, Simpore J, Musumeci S. Consumption of wheat foodstuffs not a risk for celiac disease occurrence in Burkina Faso. J Pediatr Gastroenterol Nutr. 2002;35(2):233-4.

31.Gregory C, Ashworth M, Eade OE, Holdstock G, Smith CL, Wright R. Delay in diagnosis of adult coeliac disease. Digestion. 1983;28(3):201-4.

32.Lankisch PG, Martinez Schramm A, Petersen F, Droge M, Lehnick D, Lembcke B. Diagnostic intervals for recognizing celiac disease. $Z$ Gastroenterol. 1996;34(8):473-7.

33.Pietzak M. Follow-up of patients with celiac disease: achieving compliance with treatment. Gastroenterology. 2005;128(4 Supp1 1):S135-41. 